Check for updates

Cite this: RSC Adv., 2017, 7, 47243

Received 10th August 2017

Accepted 22nd September 2017

DOI: $10.1039 / \mathrm{c} 7 \mathrm{ra0} 8828 \mathrm{k}$

rsc.li/rsc-advances

\title{
Strain-induced thermoelectric performance enhancement of monolayer $\mathrm{ZrSe}_{2}$
}

\author{
Dan Qin, (D) ab Xu-Jin Ge, ${ }^{a}$ Guang-qian Ding, ${ }^{a}$ Guo-ying Gao (D) a and Jing-Tao Lü ${ }^{\star a}$
}

Monolayer $\mathrm{ZrSe}_{2}$ was previously predicted to be one kind of excellent thermoelectric material due to its low lattice thermal conductivity. Motivated by the recent proposal of enhancing thermoelectric performance via strain-induced electronic band degeneracy, we have performed first-principles calculations on the effect of biaxial tensile strain on the thermoelectric properties of monolayer $\mathrm{ZrSe}_{2}$ combined with Boltzmann transport theory and deformation potential theory. The theoretical results demonstrate that the band degeneracy reaches its maximum at $7.5 \%$ strain, resulting in an increase of the Seebeck coefficient and, at the same time, a decrease of the lattice thermal conductivity. At this optimal strain, a two-fold increase of the figure of merit is obtained for an $\mathrm{n}$-doped $\mathrm{ZrSe}_{2}$ monolayer at room temperature. Moreover, the figures of merit for $\mathrm{p}$ - and $\mathrm{n}$-type doping are much more balanced in the strain case compared with the unstrained one.

\section{Introduction}

As a potentially important technology for the conversion of heat flow to electrical work and vice versa, thermoelectrics (TEs) have attracted more and more attention over the past several decades. ${ }^{1}$ The thermoelectric performance is usually characterized in terms of the dimensionless figure of merit $Z T$, which is given by $Z T=S^{2} \sigma T /\left(k_{\mathrm{L}}+k_{\mathrm{e}}\right)$. Here, $S$ is the thermopower or Seebeck coefficient, $\sigma$ the electrical conductivity, $T$ the absolute temperature, and $k_{\mathrm{L}}$ and $k_{\mathrm{e}}$ are the thermal conductivities contributed by a phonon and an electron, respectively. ${ }^{2}$ Therefore, large values of $Z T$ demand high $S$ and high $\sigma$, as well as low thermal conductivity $\left(k_{\mathrm{L}}+k_{\mathrm{e}}\right)$. Since an increase in $S$ normally implies a decrease in $\sigma$, while an increase in $\sigma$ implies an increase in $k_{\mathrm{e}}$ as given by the Wiedemann-Franz law, it is hard work to increase $Z T$ in typical thermoelectric materials. In the past decade, a lot of effort has been focused on low-dimensional systems for their unique electronic and phonon transport behaviors. ${ }^{3-11}$

Thermoelectric transport performance can benefit from several advantages of low-dimensional materials, including: (1) sharp changes of the density of states near the Fermi energy $E_{\mathrm{F}}$, resulting in an increase in the Seebeck coefficient; (2) opportunities to exploit the anisotropic Fermi surfaces in multi-valley semiconductors; (3) opportunities to increase the boundary scattering of phonons at interfaces, resulting in a decrease in lattice thermal conductivity. In recent years, two-dimensional

${ }^{a}$ School of Physics, Huazhong University of Science and Technology, 430074 Wuhan, P. R. China.E-mail: qindan_ok@163.com; guoying_gao@mail.hust.edu.cn; jtlu@hust. edu.cn

${ }^{b}$ Physics Department, Binzhou Medical University, 264003 Yantai, Shangdong, P. R. China
(2D) materials have been widely investigated as thermoelectric materials, such as graphene, ${ }^{12}$ boron-nitride, ${ }^{13}$ and black phosphorus. $^{14}$ Particularly, the class of transition-metal dichalcogenides (TMDCs) $\mathrm{MX}_{2}(\mathrm{M}=\mathrm{Mo}, \mathrm{W} ; \mathrm{X}=\mathrm{S} \text {, Se })^{15-19}$ has received great interest due to their unique properties being superior to those of their corresponding bulk states. Typical monolayers of $\mathrm{MoS}_{2}$ (ref. 18) and $\mathrm{WSe}_{2}$ (ref. 19) exhibit $Z T$ values of 0.11 and 0.7 at high temperature. Such low $Z T$ is mainly caused by high $k_{\mathrm{L}}$, which is as large as $100 \mathrm{~W} \mathrm{~m}^{-1} \mathrm{~K}^{-1}$ for $\mathrm{MoS}_{2}$ at room temperature and larger than $40 \mathrm{~W} \mathrm{~m}^{-1} \mathrm{~K}^{-1}$ for $\mathrm{MoSe}_{2}$. It is worth noting that all of the above explored monolayer TMDCs have hexagonal $2 \mathrm{H}$-type crystal structure. Another trigonal 1T$\mathrm{CdI}_{2}$ type of TMDC with $\mathrm{M}=\mathrm{Zr}$ and $\mathrm{Hf}$ has recently been predicted to have much lower $k_{\mathrm{L}}{ }^{20}$ with $1.2 \mathrm{~W} \mathrm{~m}^{-1} \mathrm{~K}^{-1}$ for $\mathrm{ZrSe}_{2}$ and $1.8 \mathrm{~W} \mathrm{~m}^{-1} \mathrm{~K}^{-1}$ for $\mathrm{HfSe}_{2}$ at $300 \mathrm{~K}$. Similar behavior was also found in $\mathrm{CdI}_{2}$ type monolayer $\mathrm{ZrS}_{2} \cdot{ }^{21}$ These results indicate that monolayer TMDCs with $\mathrm{CdI}_{2}$ type structure may have better thermoelectric performance due to their much lower $k_{\mathrm{L}}$ than those of $\mathrm{MoS}_{2}$ type monolayers.

Recently, the thermoelectric performance of $\mathrm{CdI}_{2}$ type monolayer $\mathrm{HfS}_{2}$ was found to be further enhanced through strain-induced electronic band degeneracy. ${ }^{22}$ It is known that the electronic transport performance depends primarily on $N_{\mathrm{V}} \mu\left(m_{\mathrm{b}}^{*} / m_{\mathrm{e}}\right)^{3 / 2},{ }^{23-25}$ where $N_{\mathrm{V}}$ is the valley degeneracy, $\mu$ is the carrier mobility, $m_{\mathrm{b}}^{*}$ is the average (single valley) density of states effective mass of the degenerate valleys, and $m_{\mathrm{e}}$ is the electron mass. It has been demonstrated that a large valley degeneracy is good for thermoelectric materials, ${ }^{26}$ and can be realized when multiple bands have band extrema around the same energy (orbital degeneracy) or when multiple carrier pockets of one band in the Brillouin zone are degenerate (valley degeneracy). ${ }^{27}$ As reported in previous works, strain engineering is an effective 
approach to tune the electronic band to achieve degeneracy. ${ }^{21,22,28-31}$

Consequently, on the one hand, the strain-induced band degeneracy increases the electronic transport performance, while on the other hand, the distortion of phonon dispersion may lower $k_{\mathrm{L}}$, both leading to an enhanced $Z T$. According to the most recent work, among the $\mathrm{CdI}_{2}$ type monolayers of $\mathrm{ZrX}_{2}$ and $\mathrm{HfX}_{2}(\mathrm{X}=\mathrm{S}, \mathrm{Se}), \mathrm{ZrSe}_{2}$ shows the lowest lattice thermal conductivity. ${ }^{20}$ Moreover, the monolayers of $\mathrm{ZrX}_{2}$ and $\mathrm{HfX}_{2}$ have similar electronic band structures and can stand $10 \%$ biaxial tensile strain. ${ }^{32}$ Motivated by these results, we apply this kind of thermoelectric optimization to a monolayer of $\mathrm{ZrSe}_{2}$. In this paper, combining first-principles calculations with the Boltzmann transport equation (BTE), we perform a comprehensive study of the electronic band structures, phonon dispersions and thermoelectric properties of monolayer $\mathrm{ZrSe}_{2}$ and compare the results to those of monolayer $\mathrm{ZrS}_{2}$.

\section{Computational details}

We first carried out first-principles electronic structure calculations using the Vienna $a b$ initio simulation package (VASP).$^{33}$ The PBE form of the generalized gradient approximation ${ }^{34}$ was used for the exchange-correlation functional. The plane-wave cutoff energy was set to $600 \mathrm{eV}$ and the Monkhorst-Pack $k$ mesh is $15 \times 15 \times 1$. Geometrical structures were relaxed until the force on each atom was less than $0.001 \mathrm{eV} \AA^{-1}$. In order to avoid interaction with periodic images, $15 \AA$ of vacuum was included in the direction perpendicular to the 2D plane.

It should be pointed out that the traditional GGA functional tends to underestimate the energy band gap, which is important for thermoelectric performance. ${ }^{35}$ Whereas, the hybrid functional tends to overestimate the band gaps of monolayer TMDCs according to previous calculations. ${ }^{32}$ Here, unless specified, we performed electronic structure calculations employing the full-potential WIEN2K $\operatorname{code}^{36}$ without considering the hybrid functional after determining the equilibrium structure. We have checked that using the Heyd-Scuseria-Ernzerhof (HSE) functional ${ }^{37}$ has little effect on the power factor. In these calculations we used $R_{\mathrm{MT}} \times K_{\max }=8.0$, a muffin-tin radius of 2.0 a.u. for both the $\mathrm{Zr}$ and Se atoms, and a $19 \times 19$ $\times 1 k$-point Monkhorst-Pack mesh. Due to $\mathrm{Zr}$ and Se being $4 \mathrm{~d}$ and 3d elements, respectively, we did not consider the spinorbit coupling in all calculations. Based on the electronic structure calculations, a more dense nonshifted $87 \times 87 \times 1 \mathrm{k}$ point Monkhorst-Pack mesh was used for the transport calculations to guarantee convergence and to obtain accurate carrier group velocities. The electrical conductivity and Seebeck coefficient are calculated from ${ }^{38}$

$$
\begin{gathered}
\sigma=e^{2} \int \Xi(\varepsilon)\left(-\frac{\partial f_{0}}{\partial \varepsilon}\right) \mathrm{d} \varepsilon \\
S=\frac{e}{\sigma T} \int \Xi(\varepsilon)(\varepsilon-\mu)\left(-\frac{\partial f_{0}}{\partial \varepsilon}\right) \mathrm{d} \varepsilon
\end{gathered}
$$

here, $\Xi$ is the transport distribution function with matrix elements of $\Xi^{\alpha, \beta}(\varepsilon)=\sum_{k} \tau_{k} \delta\left(\varepsilon-\varepsilon_{k}\right) v_{k}{ }^{\alpha} v_{k}{ }^{\beta}$, in which $v_{k}{ }^{\alpha}$ is the $\alpha$ th component of the group velocity of the carriers with wave vector $\mathbf{k}$.

Thermoelectric transport properties were calculated by solving the Boltzmann transport equations within the rigid band approximation and constant relaxation-time approximation (RBA and CRTA) as implemented in the BoltzTraP code. ${ }^{38}$ The CRTA has been successfully applied to many thermoelectric materials. ${ }^{39-41}$ The influence of doping is considered in the rigid band model, which assumes that light doping does not change the shape of the band structure, but only shifts the Fermi energy. By using the CRTA, $\tau$ is exactly cancelled out in eqn (2). Thus, from the above calculations we can obtain the Seebeck coefficient $S$ and the electrical conductivity over relaxation time as well $(\sigma / \tau)$. The electronic thermal conductivity $k_{\mathrm{e}}$ is calculated using the Wiedemann-Franz law, $k_{\mathrm{e}}=L \sigma T$, where $L$ is the Lorenz number. In this work we use $L=1.5 \times 10^{-8} \mathrm{~J}^{2} \mathrm{~K}^{-2} \mathrm{C}^{-2} .^{42}$ We have also calculated the lattice thermal conductivity $k_{\mathrm{L}}$ and related phonon quantities. The phonon frequencies were calculated using density functional perturbation theory (DFPT) as implemented in the VASP code. ${ }^{33}$ The phonon dispersion and eigenmodes were calculated by diagonalizing the dynamical matrix, which is constructed from harmonic interatomic force constants (IFCs) using the Phonopy code. ${ }^{43}$ In those calculations, the second order harmonic and third order anharmonic IFCs were calculated using a $5 \times 5 \times 1$ supercell with $2 \times 2 \times 1$ Monkhorst-Pack $k$ meshes and a $4 \times 4 \times 1$ supercell with $\Gamma$ point, respectively. We then solved the phonon Boltzmann transport equation based on an adaptive smearing approach to the conservation of energy ${ }^{\mathbf{4 4}}$ and with an iterative solution method, ${ }^{45}$ as implemented in ShengBTE. ${ }^{46}$

\section{Results and discussion}

Now we discuss the geometric and electronic structure of the $\mathrm{ZrSe}_{2}$ monolayer. For intrinsic monolayer $\mathrm{ZrSe}_{2}$, we have obtained the lattice parameters of $a=b=3.79 \AA$, which are very close to its bulk parameters and agree well with the values from previous literature. ${ }^{47}$ The top and side views of monolayer $\mathrm{ZrSe}_{2}$ are shown in Fig. 1(a) and (b), respectively. Based on the optimized structure, we calculated the electronic structure with the Brillouin zone path along $\Gamma-M-K-\Gamma$ as shown in Fig. 1(c). The calculated electronic band structure of the $\mathrm{ZrSe}_{2}$ monolayer is shown in Fig. 2(a), which indicates that the $\mathrm{ZrSe}_{2}$ monolayer is an indirect band gap semiconductor, with the valence band maximum (VBM) and conduction band minimum (CBM) located at the $\Gamma$ and $M$ points, respectively. The band gap is calculated to be $0.47 \mathrm{eV}$, which shows good agreement with previous calculations. ${ }^{\mathbf{4 7}}$

In order to increase the degenerate carrier pockets near the Fermi level, we applied a series of in-plane biaxial tensile strains to the $\mathrm{ZrSe}_{2}$ monolayer, which are defined as $\varepsilon=\left(a-a_{0}\right) / a_{0} \times$ $100 \%$. Here $a_{0}$ is an unstrained cell parameter, and $a$ is a strained cell parameter. Tensile strains ranging from 0 to $9 \%$ were considered and only the results of $6 \%, 7 \%, 7.5 \%, 8 \%$ and $9 \%$ tensile strain are given as representative cases; the corresponding band structures are shown in Fig. 2(a), (b), (c), (d), (e) and (f), respectively. The DFT calculated band gaps increase 


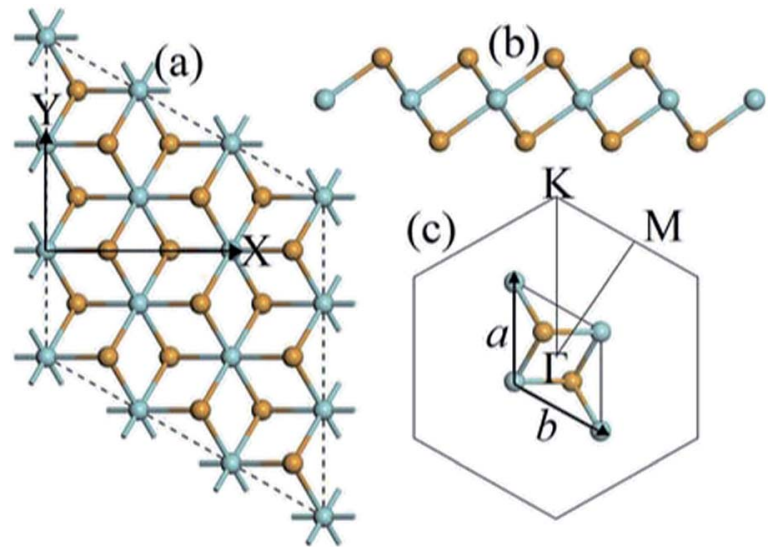

Fig. 1 (a) Top and (b) side views of monolayer $\mathrm{ZrSe}_{2}$ in $\mathrm{Cdl}_{2}$ type structure, where the green and yellow balls represent the $\mathrm{Zr}$ and Se atoms, respectively. $X$ and $Y$ denote the armchair and zigzag directions of the monolayer, respectively. (c) The unit cell and corresponding Brillouin zone path with high-symmetry points at $\Gamma(0,0,0), M(0.5,0,0)$ and $K(1 / 3,1 / 3,0)$.

from $0.47 \mathrm{eV}$ at zero strain to $1.18 \mathrm{eV}$ when the biaxial tensile strain is beyond $7.5 \%$, as shown in Fig. 3. As can be seen in Fig. 2(a), the valence band valleys near the Fermi level are denoted by I, II and III, where the extrema of valleys I and III are about $0.6 \mathrm{eV}$ below valley II. The two conduction band valleys near the Fermi level are denoted by IV and V, with two valleys being degenerate at the II and IV points. The energy difference between valley I and II (IV and V) is denoted as $\Delta_{\mathrm{V}}\left(\Delta_{\mathrm{C}}\right)$, namely $\Delta_{\mathrm{V}}=E_{\mathrm{II}}-E_{\mathrm{I}}$ and $\Delta_{\mathrm{C}}=E_{\mathrm{IV}}-E_{\mathrm{V}}$. It is suggested that the bands may be regarded as effectively converged when their energy

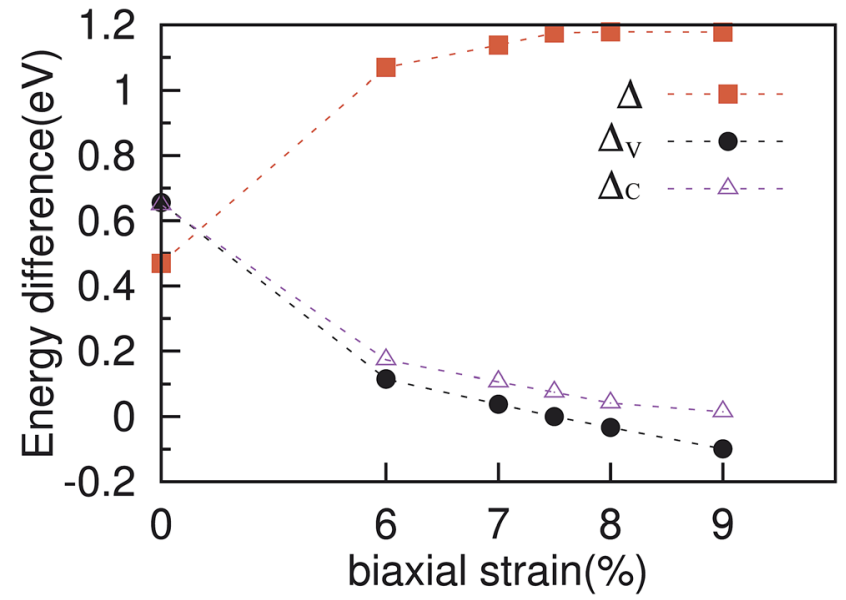

Fig. 3 Calculated electronic band gaps $(\Delta)$ and energy difference between the valence and conduction band valleys $\left(\Delta_{\mathrm{V}}\right.$ and $\left.\Delta_{\mathrm{C}}\right)$ as a function of the applied biaxial tensile strain ranging from 0 to $9 \%$.

separation is small compared with $k_{\mathrm{B}} T$, where $k_{\mathrm{B}}$ is the Boltzmann constant. ${ }^{26}$ Thus it is clear that a high valley degeneracy in the Brillouin zone can be obtained by minimizing the values of $\Delta_{\mathrm{V}}$ and/or $\Delta_{\mathrm{C}}$. At zero strain, the extreme of valley II is the highest among the three valence band valleys, while the V valley is the lowest among the two conduction valleys. When tensile strain is applied, the extremes of these valence band valleys gradually get closer to each other in energy as the strain increases, resulting in a decrease of $\Delta_{\mathrm{V}}$ as shown in Fig. 2. At a strain of $7.5 \%$, the three valleys are nearly degenerate. As a consequence, the degeneracy of the hole carrier pockets reaches the maximum. On further increasing the biaxial strain, the

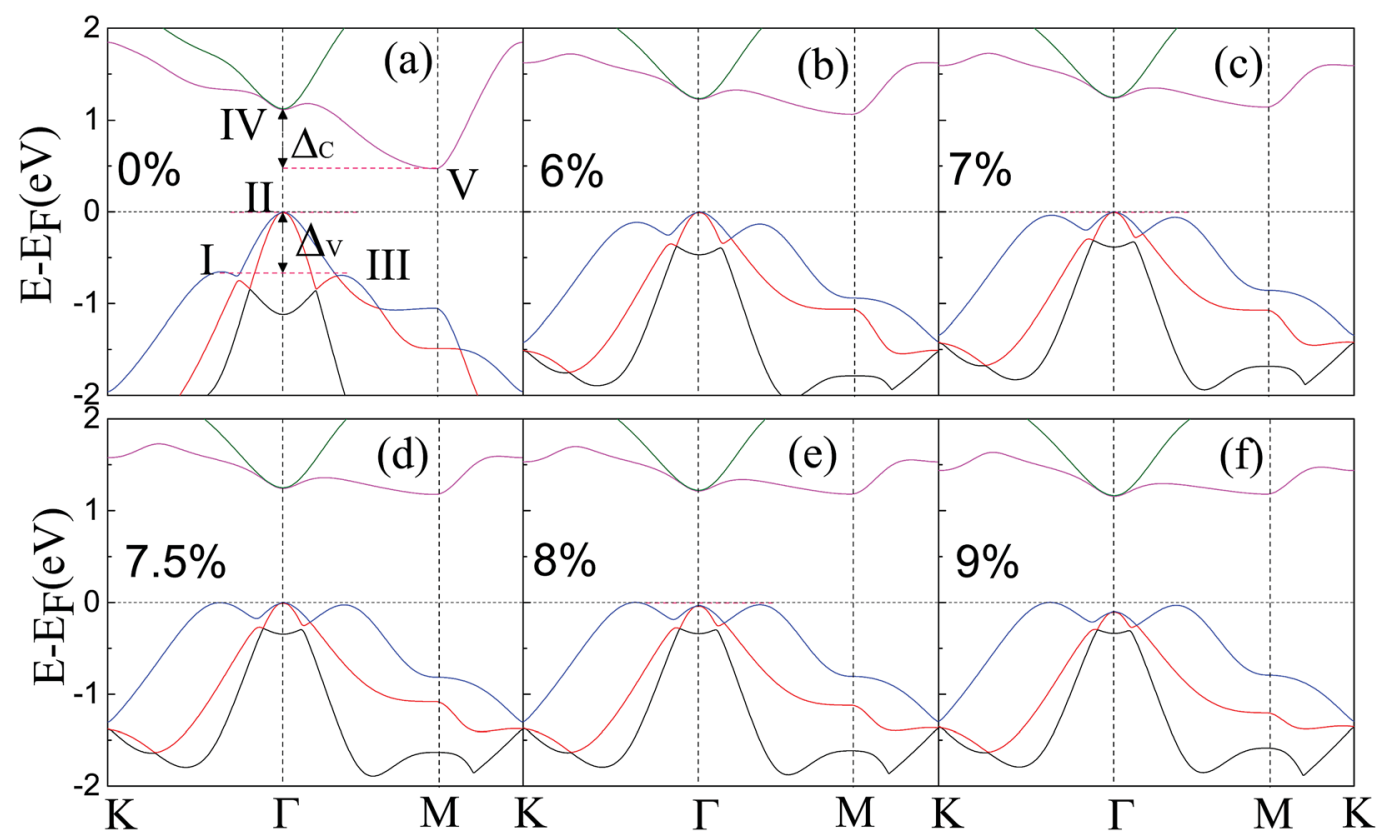

Fig. 2 Band structures of the $\mathrm{ZrSe}_{2}$ monolayer along high-symmetry $k$-points under different biaxial strains of (a) $0 \%$, (b) $6 \%$, (c) $7 \%$, (d) $7.5 \%$, (e) $8 \%$, and (f) $9 \%$. The three valence band valleys near the Fermi level are denoted by I, II, and III, respectively. The two conduction band valleys near the Fermi level are denoted by IV and V, respectively. $\Delta_{V}\left(\Delta_{C}\right)$ represents the energy difference between I and II (IV and V). 
extreme of valley II shifts down and thus the degeneracy level is reduced, as displayed in Fig. 2. For the conduction band, as demonstrated in Fig. 2, the band valley $\mathrm{V}$ is elevated gradually following the increasing strain, leading to a decrease of $\Delta_{\mathrm{C}}$. The increase of the band valley degeneracy is beneficial to the thermoelectric performance. We thus expect an enhanced thermoelectric performance for monolayer $\mathrm{ZrSe}_{2}$ via this straininduced valley degeneracy. Next we will discuss the electronic properties of $\mathrm{ZrSe}_{2}$ under different tensile strains.

\section{Electronic transport properties}

In this part, we analyse the electronic properties of the $\mathrm{ZrSe}_{2}$ monolayer without and with biaxial strain. We have performed calculations of the electronic transport coefficients by using the semiclassical Boltzmann theory.

The left (right) panels in Fig. 4 show the electronic transport coefficients with respect to the biaxial tensile strain of $0 \%, 6 \%$, $7.5 \%$ and $9 \%$ as a function of the hole (electron) concentration at $T=300 \mathrm{~K}$. As can be seen in Fig. 4(a) and (b), the electrical conductivity $\sigma / \tau$ increases with increasing carrier concentration and decreases with increasing biaxial tensile strains, which is because of the increase of the band gap as shown in Fig. 3. Such an influence on conductivity with the applied strain will exert a negative effect on the thermoelectric properties. However, the calculated absolute values of the Seebeck coefficients at $300 \mathrm{~K}$ (see Fig. 4(c) and (d)) increase with the increase of the strain initially, then reach their maxima at a strain of $7.5 \%$, and then slightly decrease when the strain is further increased. It is important to note that the Seebeck coefficients for both n- and p-type doped $\mathrm{ZrSe}_{2}$ monolayers at a biaxial tensile strain of $7.5 \%$ are quite enlarged at room temperature, reaching a peak value of $725 \mu \mathrm{V} \mathrm{K}^{-1}$ at a hole concentration around $10^{11} \mathrm{~cm}^{-2}$. These enhanced values of $S$ for a monolayer compare favorably with those reported for optimized $\mathrm{MoS}_{2}{ }^{18}$ and $\mathrm{HfO}_{2} \cdot{ }^{48}$ The trend of the Seebeck coefficient as a function of the strain is almost in accordance with the difference energy of $\Delta_{\mathrm{V}}$ (see Fig. 3), that is, the enhanced Seebeck coefficient is mainly due to the increased degeneracy of the band valley. Fig. 4(e) and (f) show the power factor (PF) $S^{2} \sigma / \tau$ under different strains at $300 \mathrm{~K}$ dependent on the carrier concentration for the $\mathrm{p}$ - and n-type carriers, respectively. The results show that the power factor for n-type doping is much larger than that for the p-type doping at zero strain, which is due to the larger Seebeck coefficient for n-type doping. In addition, for either $\mathrm{p}$ - or n-type doping, the peak value of the $\mathrm{PF}$ initially increases with increasing strain, then reaches the maximum at a strain of $7.5 \%$, and then declines slightly on increasing the strain further. It is worth noting that the trend of the PF with respect to the strain is the same as that of the Seebeck coefficient, indicating that the negative effect of the strain on the electrical conductivity is counteracted by the increase of the Seebeck coefficient.

Within the relaxation time approximation, the Seebeck coefficient can be calculated independently of the relaxation time $\tau$, but evaluation of the electrical conductivity $(\sigma)$ requires knowledge of $\tau$, which is obtained from either fitting of the experiments or theoretical estimation. There are different types of carrier scattering processes that need to be considered in

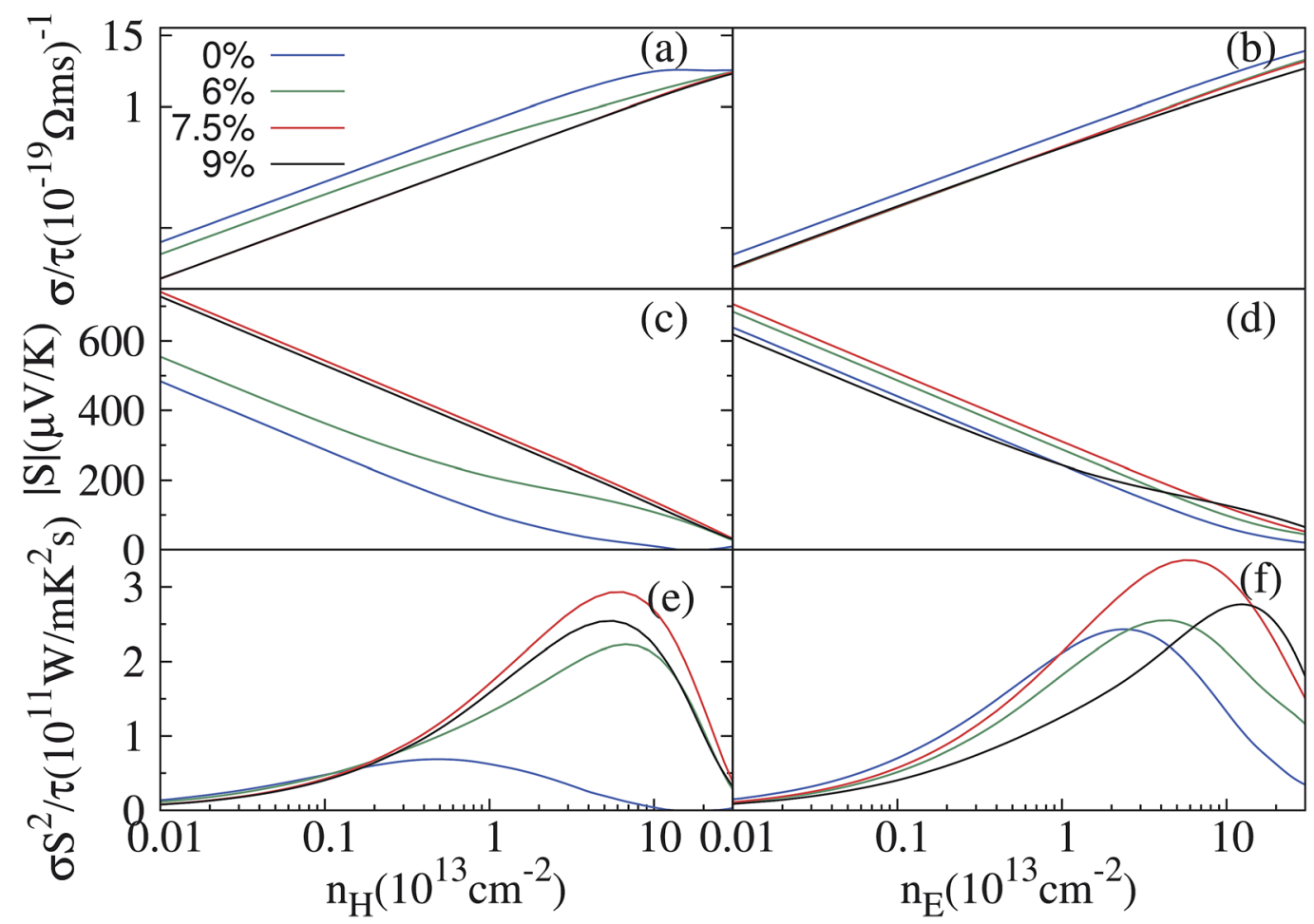

Fig. 4 Calculated transport coefficients $\sigma / \tau$ (a and b), $S$ (c and d), and $\sigma / S^{2} \tau$ (e and f) under different biaxial strains of $6 \%, 7.5 \%$ and $9 \%$ as a function of carrier concentration. The left and right panels correspond to $\mathrm{p}$ - and $\mathrm{n}$-type doping at room temperature, respectively. 
estimating the relaxation time. Here we take into account only the intrinsic scattering mechanism, namely, the interaction of electrons with acoustic phonons. We use the Bardeen-Shockley deformation potential approach for 2D materials, ${ }^{49,50}$ where the atomic displacement associated with a long-wavelength acoustic phonon leads to a deformation of the crystal, and in turn, to a shift of the electronic energy dispersion. Followed by the deformation potential theory, the carrier mobility and the relaxation time $\tau$ in $2 \mathrm{D}$ materials are calculated using the equations

$$
\begin{gathered}
\mu=\frac{e \hbar^{3} C_{2 \mathrm{D}}}{k_{\mathrm{B}} T m^{*} m_{\mathrm{d}}\left(E_{1}\right)^{2}} \\
\tau=\frac{\mu m^{*}}{e}
\end{gathered}
$$

where $m^{*}$ is the effective mass in the transport direction, $m_{\mathrm{d}}$ is the average effective mass defined by $m_{\mathrm{d}}=\sqrt{m_{x}^{*} m_{y}^{*}}, E_{1}$ is the deformation potential constant and $C_{2 \mathrm{D}}$ is the effective elastic modulus. The calculated results of these parameters are listed in Table 1. We now turn our attention to the effective mass and carrier mobility, as shown in Table 1. Under unstrained conditions, the effective mass is almost isotropic for the hole due to the isotropic band dispersion near the VBM. For the electron, the effective mass is highly anisotropic, and is much higher along the armchair direction $\left(2.32 m_{\mathrm{e}}\right)$ than along the zigzag direction $\left(0.34 \mathrm{~m}_{\mathrm{e}}\right)$, since the conduction band dispersion near the Fermi level along the $M-K$ direction (zigzag direction in real space) is much steeper than that along the $M-\Gamma$ direction (armchair direction in real space). As we know, the small effective masses of the carriers may lead to a high carrier mobility. Using the deformation potential theory, the charge carrier mobility can be predicted from the carrier effective mass, deformation potential constant and effective 2D elastic modulus, as listed in Table 1. When a biaxial strain of $7.5 \%$ is applied, the effective mass for an electron increases in the zigzag direction, but decreases significantly in the armchair direction. It can be seen from the results that the carrier mobility is reduced under strain, resulting in the decrease of the electrical conductivity $(\sigma / \tau)$. This is in agreement with the electronic transport calculation as shown in Fig. 4(a) and (b). Since the difference of carrier mobility is reduced, the power factors for $\mathrm{p}$ - and n-type have just little differences (see Fig. 4(e) and (f)). Based on eqn (3) and (4), the relaxation time $\tau$ can be calculated and, as shown in Table 1, it is almost isotropic in different directions. Therefore, we will use the averaged $\tau$ of the armchair and zigzag directions to evaluate the thermoelectric performance later.

\section{Lattice thermal conductivity}

We now investigate the influence of biaxial strain on phonon dispersions and thermal conductivity. The phonon dispersions of the $\mathrm{ZrSe}_{2}$ monolayer under no strain and when under $7.5 \%$ biaxial tensile strain are displayed in Fig. 5(a) and denoted by a black solid line and red dashed line, respectively. No imaginary frequency is observed, indicating kinetic stability. By means of the phonon Boltzmann transport equation and DFT, the lattice thermal conductivity is calculated, as depicted in Fig. 5(b). It can be seen from the figure that due to the effective strain, the phonon thermal conductivity $k_{\mathrm{L}}$ has been successfully reduced. The reduced lattice thermal conductivity is beneficial to thermoelectric transport, which can also be achieved in $\mathrm{Mg}_{2} \mathrm{Sn}$ using strain. ${ }^{51}$ Moreover, with the increasing temperature $k_{\mathrm{L}}$ decreases following a $T^{-1}$ dependence either under no strain or under strain, as presented by the fitted lines in the figure. This suggests that Umklapp phonon scattering dominates three-phonon interactions. ${ }^{52}$ We will refer to the corresponding phonon dispersions (see Fig. 5(a)) to investigate the origin of the reduction in the thermal conductivity by applying strain. Since the heat transfer is mainly contributed to by the acoustic modes, we will focus on the three phonon models of the transverse (ZA and TA) and longitudinal acoustic (LA) modes. From Fig. 5(a), two out of three of the acoustic branches correspond to vibration within the plane of the transverse and longitudinal acoustic modes (TA and LA) at 7.5\% and are actually softer than those at 0 strain along the whole high symmetry line in the Brillouin zone. For example, the boundary frequencies of TA (LA) along $\Gamma-K$ and $\Gamma-M$ decline from 3.67 (3.67) and 1.86 (2.79) THz to 2.92 (2.92) and 1.52 (2.27) $\mathrm{THz}$, respectively, which has been proven to be beneficial to reduce the lattice thermal conductivity as reported in previous works. $^{21,53}$

To make a direct comparison, we have plotted the lattice thermal conductivities of $\mathrm{ZrSe}_{2}$ and $\mathrm{ZrS}_{2}$ (ref. 21) monolayers under no strain and under their optimal strains in Fig. 5(b).

Table 1 Effective mass $\left(m^{*}\right)$, average effective mass $\left(m_{\mathrm{d}}\right)$, elastic modulus $D$, DP constant $E_{1}$, carrier mobility $(\mu)$, and relaxation time $(\tau)$ at $300 \mathrm{~K}$ along the zigzag and armchair directions of the unstrained and $7.5 \%$ biaxial strained $\mathrm{ZrSe}_{2}$ monolayers

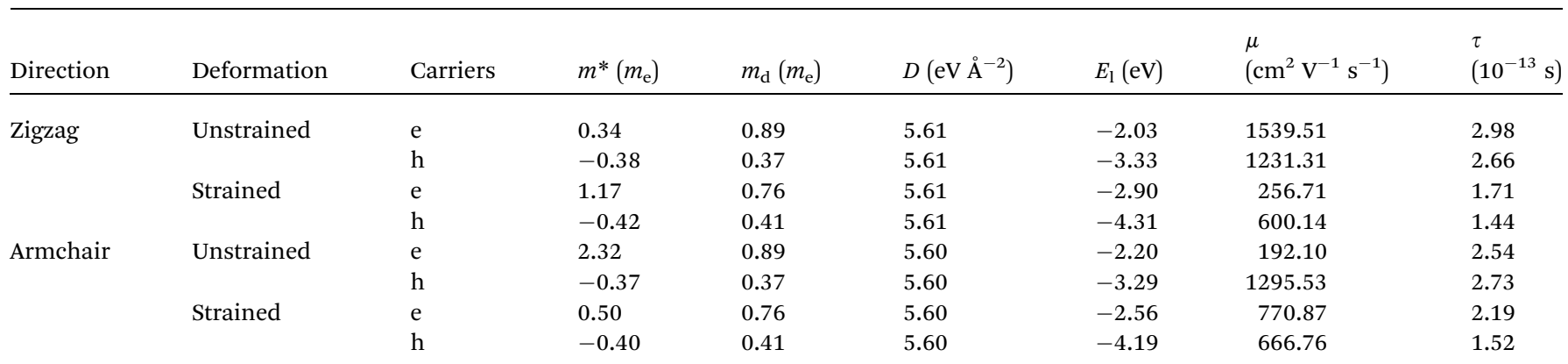



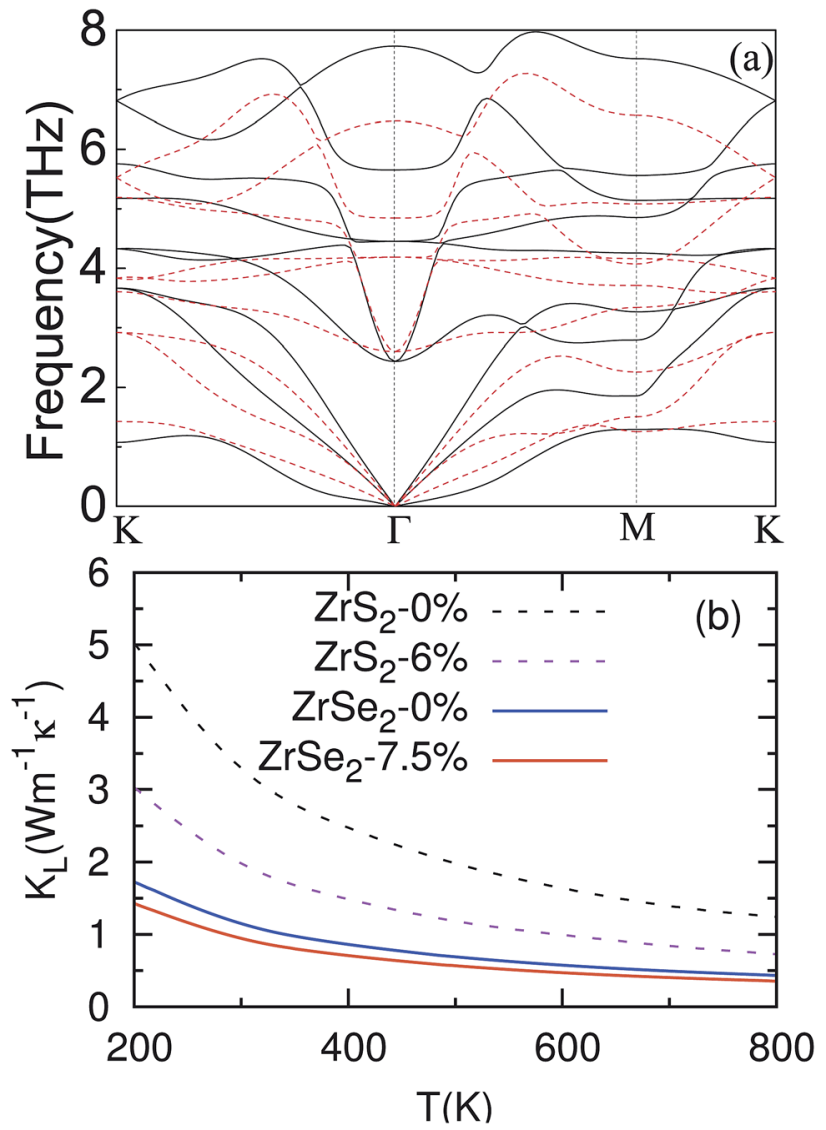

Fig. 5 (a) Calculated phonon dispersion under 0\% (denoted by the black solid line) and 7.5\% (denoted by the dashed line) strain. (b) Lattice thermal conductivity comparison of $\mathrm{ZrS}_{2}$ (under $0 \%$ and $6 \%$ strain) and $\mathrm{ZrSe}_{2}$ (under $0 \%$ and $7.5 \%$ strain) monolayers from $200 \mathrm{~K}$ to $800 \mathrm{~K}$.

Obviously, for both $\mathrm{ZrSe}_{2}$ and $\mathrm{ZrS}_{2}$ monolayers, the lattice thermal conductivity $k_{\mathrm{L}}$ decreases with the increasing temperature. Moreover, the $k_{\mathrm{L}}$ for $\mathrm{ZrSe}_{2}$ is always much lower than that of $\mathrm{ZrS}_{2}$ within the temperature range we investigated. Particularly, the $k_{\mathrm{L}}$ values at $300 \mathrm{~K}$ are $3.29(1.99) \mathrm{W} \mathrm{m}^{-1} \mathrm{~K}^{-1}$ for $\mathrm{ZrS}_{2}$ and $1.15(0.94) \mathrm{W} \mathrm{m}^{-1} \mathrm{~K}^{-1}$ for $\mathrm{ZrSe}_{2}$ under no strain (under optimal strain), which implies that $\mathrm{ZrSe}_{2}$ may be preferable to $\mathrm{ZrS}_{2}$ in thermoelectric applications.

\section{Figure of merit}

Now we are in a position to evaluate the thermoelectric figure of merit $Z T$. The doping-dependent $Z T$ for unstrained and $7.5 \%$ strained $\mathrm{ZrSe}_{2}$ monolayers at room temperature are depicted in Fig. 6. For the unstrained case, the maximum $Z T$ value of the ntype doped system is 4.26 , which is about 2 times larger than that of the p-type system. When a strain of $7.5 \%$ is applied, the values of $Z T$ for the n-type and p-type doped systems increase to 4.58 and 3.84, respectively. The enhanced $Z T$ is mainly due to the increased power factor $S^{2} \sigma$ as well as the decreased lattice thermal conductivity $k_{\mathrm{L}}$ when under strain. Furthermore, the strain engineering has induced much more balanced $Z T$ values

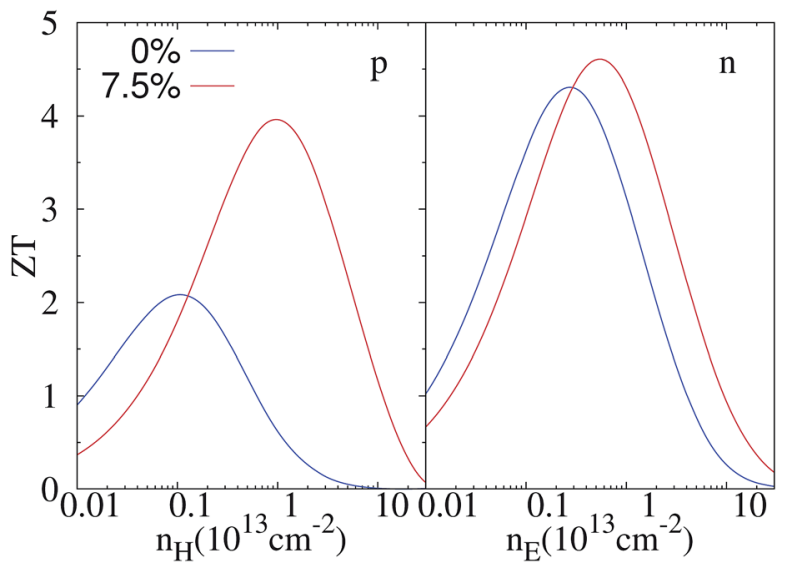

Fig. 6 Estimated figure of merit of monolayer $\mathrm{ZrSe}_{2}$ for both $\mathrm{p}$ - (left panel) and n-type (right panel) under $0 \%$ and $7.5 \%$ strain at $300 \mathrm{~K}$.

for n- and p-type, which are very desirable for the fabrication of thermoelectric moduli.

In addition, we should mention some facts as follows: (1) to date, developing an effective method to apply tunable strain in 2D layers such as $\mathrm{MoS}_{2}$ is highly desirable. Based on the wealth of theoretical studies on band engineering using strain and some strategies for exploring strain on 2D materials in recent experiments, such as using piezoelectric stretching ${ }^{54}$ and exploiting the thermal expansion mismatch, ${ }^{55,56}$ it is feasible to realize a thermoelectric response on a $\mathrm{ZrSe}_{2}$ monolayer under strain experimentally. (2) Achieving the maximum available $Z T$ requires a certain (optimal) doping level. In principle, the optimal carrier concentration should be used to produce fully optimized thermoelectric materials to ensure peak performance for thermoelectric applications. For example, by alloying PbTe with MgTe properly, ZT can reach 1.7. ${ }^{57}$ (3) The results of this work, together with the recent reports on monolayer $\mathrm{ZeS}_{2}{ }^{21}$ and $\mathrm{HfS}_{2},{ }^{22}$ pave the way for further enhancement of the thermoelectric performance of $\mathrm{CdI}_{2}$ type monolayer TMDCs.

Finally, we would like to check the influence of the HSE functional and spin-orbit coupling (SOC) on the band structure and electronic transport properties. The calculated band structures and power factors (at $300 \mathrm{~K}$ ) of monolayer $\mathrm{ZrSe}_{2}$ without strain using the HSE functional and including the SOC effect are shown in Fig. 7, in comparison to the normal GGA functional. Firstly, we concentrate on the influence of the HSE functional. One can see that the HSE functional predicts a band gap of $0.53 \mathrm{eV}$, being a little larger than $0.47 \mathrm{eV}$ from GGA, indicating a small dependence of the band structure on the functionals used. But, we find that the power factor from HSE and GGA for both the n-type and p-type systems shows little difference. Hence, the predictions in this work do not depend on the functionals used.

With regard to the SOC effect, the difference of band structure lies in the uppermost two valence bands at the $\Gamma$ point. They are degenerate at $\Gamma$ without SOC, while they split into two individual bands when considering the SOC effect. This influence of SOC is similar to the situation in the bulk systems. ${ }^{58}$ 

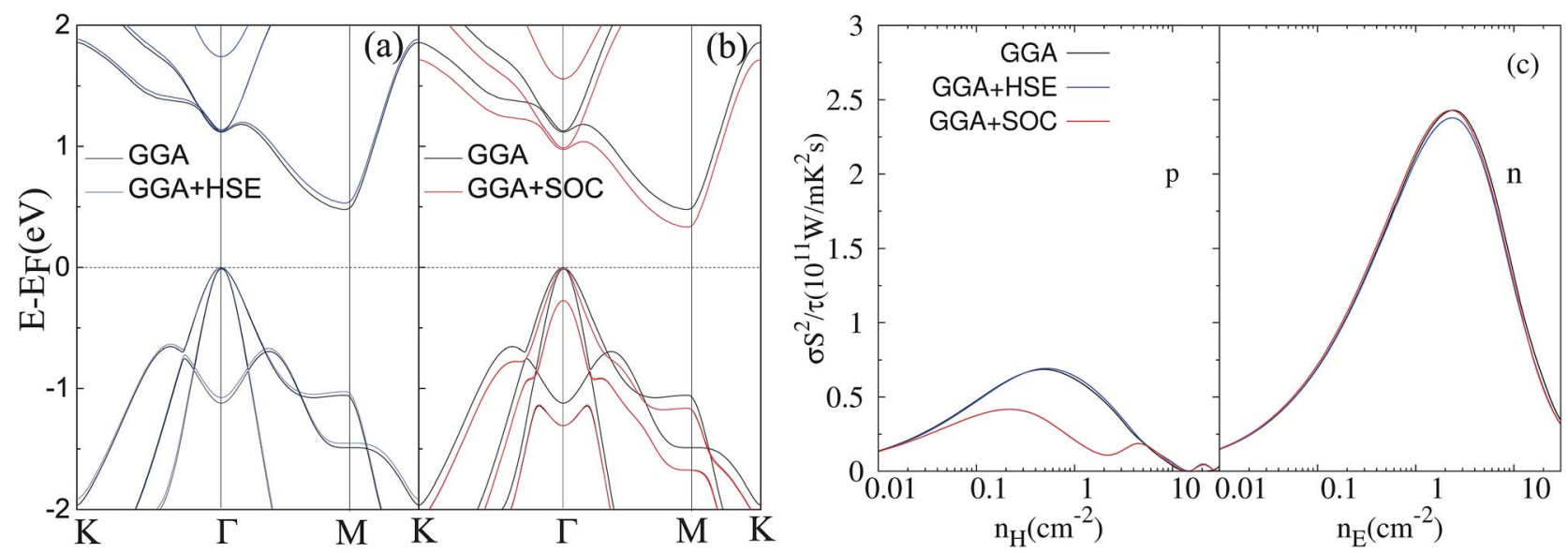

Fig. 7 Calculated band structures of the $\mathrm{ZrSe}$, monolayer using the HSE hybrid functional (a) and SOC effect (b), also with a comparison to the normal GGA functional. (c) A comparison of power factor at room temperature predicted from different methods of GGA, HSE and SOC.

Regarding the power factor, we find that there is little difference for n-type, while the p-type power factor decreases when considering the SOC effect. The decrease of the p-type power factor is due to the decreased band degenerate level of the two uppermost valence bands at the $\Gamma$ point. Overall, the HSE functional has little influence on the thermoelectric transport performance, while the SOC effect may influence the p-type thermoelectric performance but it should not influence the strain dependence of the thermoelectric properties, which is the focus of this work.

\section{Conclusions}

In summary, based on first-principles calculations combined with Boltzmann equations, we have investigated the electrical, thermal and thermoelectric properties of monolayer $\mathrm{ZrSe}_{2}$ with and without biaxial tensile strain. The results reveal that at a strain of $7.5 \%$ the three valence band valleys converge in energy and the degeneracy of the valleys reaches a maximum, resulting in the maximum power factor $\left(S^{2} \sigma\right)$. Thus we obtain a maximum $Z T$ of 4.58 and 3.84 for $\mathrm{p}$ - and n-type doping at room temperature, respectively, which are larger than those of the case without strain. Furthermore, these results verify our expectation that at the optimal strain, the values of $Z T$ for monolayer $\mathrm{ZrSe}_{2}$ are larger than those of $\mathrm{ZrS}_{2}$ (2.4 for p-type and 1.8 for $\mathrm{n}$-type doping) ${ }^{21}$ and $\mathrm{HfS}_{2}$ (3.67 for p-type and 3.08 for $\mathrm{n}$ type doping). ${ }^{22}$ Above all, our studies indicate that the thermoelectric performance of a $\mathrm{ZrSe}_{2}$ monolayer can be effectively enhanced by band valley engineering through the application of biaxial strain. We can further conclude that at the optimal strain, the monolayer $\mathrm{ZrSe}_{2}$ is preferable to $\mathrm{ZrS}_{2}$ and $\mathrm{HfS}_{2}$ for thermoelectric transport due to the lower lattice thermal conductivity.

\section{Conflicts of interest}

There are no conflicts to declare.

\section{Acknowledgements}

The authors gratefully acknowledge the financial support from the National Natural Science Foundation of China (Grant No. 6137105 and Grant No. 51401031).

\section{References}

1 L. E. Bell, Science, 2008, 321, 1457-1461.

2 J. Sootsman, D. Chung and M. Kanatzidis, Angew. Chem., Int. Ed., 2009, 48, 8616-8639.

3 L. D. Hicks and M. S. Dresselhaus, Phys. Rev. B: Condens. Matter Mater. Phys., 1993, 47, 12727-12731.

4 L. Hicks and M. Dresselhaus, Phys. Rev. B: Condens. Matter Mater. Phys., 1993, 47, 16631.

5 G. Mahan and J. Sofo, Proc. Natl. Acad. Sci. U. S. A., 1996, 93, 7436-7439.

6 M. S. Dresselhaus, G. Chen, M. Y. Tang, R. Yang, H. Lee, D. Wang, Z. Ren, J.-P. Fleurial and P. Gogna, Adv. Mater., 2007, 19, 1043-1053.

7 G. J. Snyder and E. S. Toberer, Nat. Mater., 2008, 7, 105-114. 8 A. I. Hochbaum, R. Chen, R. D. Delgado, W. Liang, E. C. Garnett, M. Najarian, A. Majumdar and P. Yang, Nature, 2008, 451, 163-167.

9 Y. Dubi and M. Di Ventra, Rev. Mod. Phys., 2011, 83, 131.

10 X.-Y. Mi, X. Yu, K.-L. Yao, X. Huang, N. Yang and J.-T. LuİL', Nano Lett., 2015, 15, 5229-5234.

11 Y. M. Brovman, J. P. Small, Y. Hu, Y. Fang, C. M. Lieber and P. Kim, J. Appl. Phys., 2016, 119, 234304.

12 Y. Anno, Y. Imakita, K. Takei, S. Akita and T. Arie, 2D Mater., 2017, 4, 025019.

13 A. Tabarraei, Comput. Mater. Sci., 2015, 108, 66-71.

14 R. Fei, A. Faghaninia, R. Soklaski, J.-A. Yan, C. Lo and L. Yang, Nano Lett., 2014, 14, 6393-6399.

15 W. Huang, H. Da and G. Liang, J. Appl. Phys., 2013, 113, 104304. 
$16 \mathrm{~J}$. Maassen and M. S. Lundstrom, Nanotechnology (IEEENANO), 2014 IEEE 14th International Conference on, 2014, pp. 904-907.

17 K. Ghosh and U. Singisetti, J. Appl. Phys., 2015, 118, 135711.

18 Z. Jin, Q. Liao, H. Fang, Z. Liu, W. Liu, Z. Ding, T. Luo and N. Yang, Sci. Rep., 2016, 5, 18342.

19 S. Kumar and U. SchwingenschlÂügl, Chem. Mater., 2015, 27, 1278-1284.

20 G. Ding, G. Y. Gao, Z. Huang, W. Zhang and K. Yao, Nanotechnology, 2016, 27, 375703.

21 H. Y. Lv, W. J. Lu, D. F. Shao, H. Y. Lu and Y. P. Sun, J. Mater. Chem. C, 2016, 4, 4538-4545.

22 H. Lv, W. Lu, X. Luo, H. Lu, X. Zhu and Y. Sun, arXiv preprint arXiv:1608.05464, 2016.

23 H. Goldsmid, Thermoelectric refrigeration, The International cryogenics monograph series, 1964.

24 D. M. Rowe, CRC handbook of thermoelectrics, CRC press, 1995.

25 F. J. DiSalvo, Science, 1999, 285, 703-706.

26 Y. Pei, X. Shi, A. LaLonde, H. Wang, L. Chen and G. J. Snyder, Nature, 2011, 473, 66-69.

27 Y. Pei, H. Wang and G. Snyder, Adv. Mater., 2012, 24, 61256135.

28 S.-D. Guo, J. Mater. Chem. C, 2016, 4, 9366-9374.

29 G. Zhang and Y.-W. Zhang, Mech. Mater., 2015, 91, 382-398.

30 R. Roldán, A. Castellanos-Gomez, E. Cappelluti and F. Guinea, J. Phys.: Condens. Matter, 2015, 27, 313201.

31 H. Balout, P. Boulet and M.-C. Record, J. Electron. Mater., 2013, 42, 3458-3466.

32 H. Guo, N. Lu, L. Wang, X. Wu and X. C. Zeng, J. Phys. Chem. $C, 2014$, 118, 7242-7249.

33 G. Kresse and J. Furthmüller, Phys. Rev. B: Condens. Matter Mater. Phys., 1996, 54, 11169.

34 J. P. Perdew, K. Burke and M. Ernzerhof, Phys. Rev. Lett., 1996, 77, 3865-3868.

35 Y.-S. Kim, M. Marsman, G. Kresse, F. Tran and P. Blaha, Phys. Rev. B: Condens. Matter Mater. Phys., 2010, 82, 205212.

36 P. Blaha, K. Schwarz, G. Madsen, D. Kvasnicka and J. Luitz, WIEN2k An augmented plane wave+ local orbitals program for calculating crystal properties, 2001.

37 J. Heyd, G. E. Scuseria and M. Ernzerhof, J. Chem. Phys., 2003, 118, 8207-8215.

38 G. K. Madsen and D. J. Singh, Comput. Phys. Commun., 2006, 175, 67-71.
39 L. Xi, Y. B. Zhang, X. Y. Shi, J. Yang, X. Shi, L. D. Chen, W. Zhang, J. Yang and D. J. Singh, Phys. Rev. B: Condens. Matter Mater. Phys., 2012, 86, 155201.

40 D. Parker and D. J. Singh, Phys. Rev. X, 2011, 1, 021005.

41 H. Peng, N. Kioussis and G. J. Snyder, Phys. Rev. B: Condens. Matter Mater. Phys., 2014, 89, 195206.

42 H.-S. Kim, Z. M. Gibbs, Y. Tang, H. Wang and G. J. Snyder, APL Mater., 2015, 3, 041506.

43 A. Togo, F. Oba and I. Tanaka, Phys. Rev. B: Condens. Matter Mater. Phys., 2008, 78, 134106.

44 W. Li, N. Mingo, L. Lindsay, D. A. Broido, D. A. Stewart and N. A. Katcho, Phys. Rev. B: Condens. Matter Mater. Phys., 2012, 85, 195436.

45 W. Li, L. Lindsay, D. Broido, D. A. Stewart and N. Mingo, Phys. Rev. B: Condens. Matter Mater. Phys., 2012, 86, 174307.

46 W. Li, J. Carrete, N. A. Katcho and N. Mingo, Comput. Phys. Commun., 2014, 185, 1747-1758.

47 H. Guo, N. Lu, L. Wang, X. Wu and X. C. Zeng, J. Phys. Chem. C, 2014, 118, 7242-7249.

48 K. Ghosh and U. Singisetti, J. Appl. Phys., 2015, 118, 135711. 49 F. Beleznay, F. Bogár and J. Ladik, J. Chem. Phys., 2003, 119, 5690-5695.

50 L.-C. Zhang, G. Qin, W.-Z. Fang, H.-J. Cui, Q.-R. Zheng, Q.-B. Yan and G. Su, Sci. Rep., 2016, 6, 19830.

51 S.-D. Guo and J.-L. Wang, RSC Adv., 2016, 6, 31272-31276.

52 A. Ward, D. A. Broido, D. A. Stewart and G. Deinzer, Phys. Rev. B: Condens. Matter Mater. Phys., 2009, 80, 125203.

53 X. J. Tan, G. Q. Liu, H. Z. Shao, J. T. Xu, B. Yu, H. C. Jiang and J. Jiang, Appl. Phys. Lett., 2017, 110, 143903.

54 Y. Y. Hui, X. Liu, W. Jie, N. Y. Chan, J. Hao, Y.-T. Hsu, L.-J. Li, W. Guo and S. P. Lau, ACS Nano, 2013, 7, 7126-7131.

55 G. Plechinger, A. Castellanos-Gomez, M. Buscema, H. S. van der Zant, G. A. Steele, A. Kuc, T. Heine, C. Schüller and T. Korn, 2D Mater., 2015, 2, 015006.

56 R. Frisenda, M. Drüppel, R. Schmidt, S. M. de Vasconcellos, D. P. de Lara, R. Bratschitsch, M. Rohlfing and A. Castellanos-Gomez, arXiv preprint arXiv:1703.02831, 2017.

57 Y. Pei, A. D. LaLonde, N. A. Heinz, X. Shi, S. Iwanaga, H. Wang, L. Chen and G. J. Snyder, Adv. Mater., 2011, 23, 5674-5678.

58 G. Yumnam, T. Pandey and A. K. Singh, J. Chem. Phys., 2015, 143, 234704. 